\title{
Accuracy of Expressions for the Magnetic Field of a Ring Head
}

\author{
B. K. Middleton, Member, IEEE, M. M. Aziz, and J. J. Miles, Member, IEEE
}

\begin{abstract}
The Fourier method used by Fan has produced a representation of the field of a semi-infinite pole head consisting of a simple analytical approximation (Karlqvist field) plus an infinite series of correction terms. A method is developed in this paper by which other approximations to head fields have their infinite series of correction terms evaluated to make them exact. Expressions with only one correction term produced by Ruigrok and Szczech et al. are studied, and enhancement of them is shown to offer accurate approximations to the exact head fields.
\end{abstract}

Index Terms-Magnetic recording, recording heads.

\section{INTRODUCTION}

$\mathbf{T}$ HE MAGNETIC potential produced by a record head with infinitely wide and deep pole faces has been calculated exactly by Fan [1]. When the poles have infinite permeability, they become equipotential surfaces with the potential variation along the faces $(y=0)$ and across the gap given by

$\varphi(x, 0)= \begin{cases}-V_{o}, & x \leq-g / 2 \\ \frac{2 V_{o}}{g} x+\sum_{n=1}^{\infty} A_{n} & \\ \cdot \sin \left(\frac{2 n \pi}{g} x\right), & -g / 2<x<g / 2 \\ V_{o}, & x \geq g / 2\end{cases}$

where $g$ is the gap length, $V_{o}=N I / 2$ where $N$ is the head coil turns and $I$ is the write current. The normalized coefficients $A_{n} / V_{o}$ in (1) were determined exactly in [2] and are given in Table I. The first term in (1b), which represents a linear variation of the potential between the pole pieces, was used by Karlqvist [3] to produce a widely used approximation to the field below the head. In this sense, (1b) represents an approximation to the exact potential variation to which is added a series of correction terms.

In this paper, better analytical approximations than the linear one, which lead to closed form expressions for the fields below the heads, are considered and a method is produced that allows the determination of the corresponding correction terms. The magnitudes of the correction terms are used to gauge the accuracy of the approximations and to further improve their accuracies. The result is simple expressions that approximate sufficiently closely to the head fields for most if not all practical purposes.

Manuscript received January 4, 2000; revised March 21, 2000. This work was supported by the Engineering and Physical Sciences Research Council.

The authors are with the Department of Computer Science, University of Manchester, Manchester M13 9PL, U.K. (e-mail: b.middleton@cs.man.ac.uk).

Publisher Item Identifier S 0018-9464(00)05844-1.
TABLE I

HARMONIC COEFFICIENT VALUES FOR DIFFERENT VALUES OF $f$ FOR THE RUIGROK APPROXIMATION

\begin{tabular}{c|c|c|c|c}
\hline $\mathbf{n}$ & $\mathbf{A}_{\mathbf{n}} / \mathbf{V}_{\mathbf{0}}$ & $\begin{array}{c}\mathbf{C}_{\mathbf{n}} / \mathbf{V}_{\mathbf{o}} \\
\mathbf{f}=\mathbf{0 . 5}\end{array}$ & $\begin{array}{c}\mathbf{C}_{\mathbf{n}} / \mathbf{V}_{\mathbf{0}} \\
\mathbf{f}=\mathbf{0 . 5 5 5 1 7 2 5 1 3}\end{array}$ & $\begin{array}{c}\mathbf{C}_{\mathbf{n}} / \mathbf{V}_{\mathbf{0}} \\
\mathbf{f}=\mathbf{0 . 6 6 2 6 5 2 4 9 5}\end{array}$ \\
\hline 1 & -0.08615711721 & 0.01068617573 & 0.00000000000 & -0.02081743072 \\
\hline 2 & 0.02915024465 & -0.00590791419 & -0.00203942074 & 0.00549667982 \\
\hline 3 & -0.01525421892 & 0.00397291346 & 0.00185129504 & -0.00228176865 \\
\hline 4 & 0.00959249954 & -0.00294154050 & -0.00155847153 & 0.00113584527 \\
\hline 5 & -0.00668032954 & 0.00230759905 & 0.00131582583 & -0.00061621897 \\
\hline 6 & 0.00496516208 & -0.00188187028 & -0.00112633432 & 0.00034550351 \\
\hline 7 & -0.00386089655 & 0.00157808099 & 0.00097791687 & -0.00019124555 \\
\hline 8 & 0.00310357777 & -0.00135148081 & -0.00085988726 & 0.00009777197 \\
\hline 9 & -0.00255909083 & 0.00117663802 & 0.00076441892 & -0.00003861322 \\
\hline 10 & 0.00215301133 & -0.00103807694 & -0.00068595622 & 0.00000000000 \\
\hline 11 & -0.00184115674 & 0.00092586524 & 0.00062053813 & 0.00002573918 \\
\hline 12 & 0.00159585983 & -0.00083335012 & -0.00056529888 & -0.00004311600 \\
\hline 13 & -0.00139902807 & 0.00075591636 & 0.00051812896 & 0.00005490218 \\
\hline 14 & 0.00123839761 & -0.00069026700 & -0.00047744846 & -0.00006286278 \\
\hline 15 & -0.00110540014 & 0.00063398742 & 0.00044205465 & 0.00006815598 \\
\hline 16 & 0.00099389255 & -0.00058527087 & -0.00041101804 & -0.00007156113 \\
\hline 17 & -0.00089937094 & 0.00054274007 & 0.00038361029 & 0.00007361416 \\
\hline 18 & 0.00081846814 & -0.00050532779 & -0.00035925350 & -0.00007469037 \\
\hline 19 & -0.00074862316 & 0.00047219502 & 0.00033748381 & 0.00007505678 \\
\hline 20 & 0.00068785821 & -0.00044267358 & -0.00031792502 & -0.00007490595 \\
\hline & & & &
\end{tabular}

\section{THE RUIGROK APPROXIMATION}

Ruigrok [4] has suggested an approximation to the exact head field in a semi-infinite pole head. It consists of half of the Karlqvist head field plus half of that produced by a thin head for which an exact derivation is possible [5], [6]. Translating that proposition into a potential variation along $y=0$ gives

$$
\varphi(x, 0) \approx(f) \frac{2 V_{o}}{g} x+(1-f) \frac{2 V_{o}}{\pi} \sin ^{-1}\left(\frac{2 x}{g}\right)
$$

where $f$ has been introduced for reasons that will become clear later, but in this instance takes the value of $1 / 2$. To make $\varphi(x, 0)$ exact, correction terms with coefficients $C_{n}$ are added in the style of (1b) to give

$$
\begin{aligned}
\varphi(x, 0)= & (f) \frac{2 V_{o}}{g} x+(1-f) \frac{2 V_{o}}{\pi} \sin ^{-1}\left(\frac{2 x}{g}\right) \\
& +\sum_{n=1}^{\infty} C_{n} \sin \left(\frac{2 n \pi}{g} x\right) .
\end{aligned}
$$


From Mallinson [5]

$$
\begin{gathered}
\frac{2}{\pi} \sin ^{-1}\left(\frac{2 x}{g}\right)=\frac{2}{g} x+\sum_{n=1}^{\infty} A_{n}^{1} \sin \left(\frac{2 n \pi}{g} x\right) \\
|x| \leq|g / 2|
\end{gathered}
$$

where the coefficients $A_{n}^{1}$ are given exactly by [5]

$$
A_{n}^{1}=\frac{2}{n \pi} J_{o}(n \pi)
$$

and $J_{O}$ is the Bessel function of the first kind. Substitution of (4) into (3) yields

$$
\varphi(x, 0)=\frac{2 V_{o}}{g} x+\sum_{n=1}^{\infty}\left[V_{o}(1-f) A_{n}^{1}+C_{n}\right] \sin \left(\frac{2 n \pi}{g} x\right) .
$$

By matching (1b) and (6), it can be shown that

$$
\frac{C_{n}}{V_{o}}=\frac{A_{n}}{V_{o}}-(1-f) A_{n}^{1} \text {. }
$$

Table I shows the values of $A_{n} / V_{o}$ and $C_{n} / V_{o}$ for $n=1$ to $n=20$ for three different values of $f$. The value of 0.5 is that suggested by Ruigrok [4], and this leads to a reduction in the magnitudes of all of the correction terms when they are compared with those of Fan. Clearly, the Ruigrok approximation $(f=0.5)$ is significantly closer to the exact field than is the Karlqvist expression and its improved accuracy is thus verified. The other two trial values of $f$ are chosen to make $C_{1}=0$ and $C_{10}=0$, respectively, and this is done by setting $C_{n}$ to zero and solving (7) for $f$. The correction terms for $f=0.555 \cdots$ are smaller than for $f=0.5$, and therefore, the corresponding potentials and fields are even more accurate. This is indicated in Fig. 1, where the potential in the gap region according to (2) with $f=0.555$ is compared to the exact potential variation according to Fan (1b). The other value of $f$, in Table I, is used as an example to illustrate that the values of $f$ can be selected to make any particular coefficient $C_{n}$ equal to zero should that be required.

\section{THE SZCZECH et al. APPROXIMATION}

Szczech et al. [7], [8] have proposed an alternative approximation for the fields of a head following experiments on a largescale experimental model [7]. The corresponding head surface potential is

$$
\begin{gathered}
\varphi(x, 0) \approx \frac{2 K S V_{o}}{g} x+\frac{2 L S V_{o}}{M} \tanh ^{-1}\left(\frac{x}{M g}\right) \\
-g / 2 \leq x \leq g / 2
\end{gathered}
$$

where $K, L$, and $M$ are constants obtained by fitting and these were found to be equal to $0.835,0.0433$, and 0.512 , respectively. $S$ is a scale factor to cater for the difference between the surface field, as defined by Szczech et al. $\left(H_{s}=H_{x}(0,0)\right)$ and the deep-gap field $\left(H_{g}=N I / g\right)$. Using the Fourier sine series expansion of $\tanh ^{-1}$, (8) can be written as

$$
\begin{gathered}
\varphi(x, 0) \approx \frac{2 K S V_{o}}{g} x+\frac{2 L S V_{o}}{M} \sum_{n=1}^{\infty} B_{n} \sin \left(\frac{2 n \pi}{g} x\right) \\
-g / 2 \leq x \leq g / 2
\end{gathered}
$$

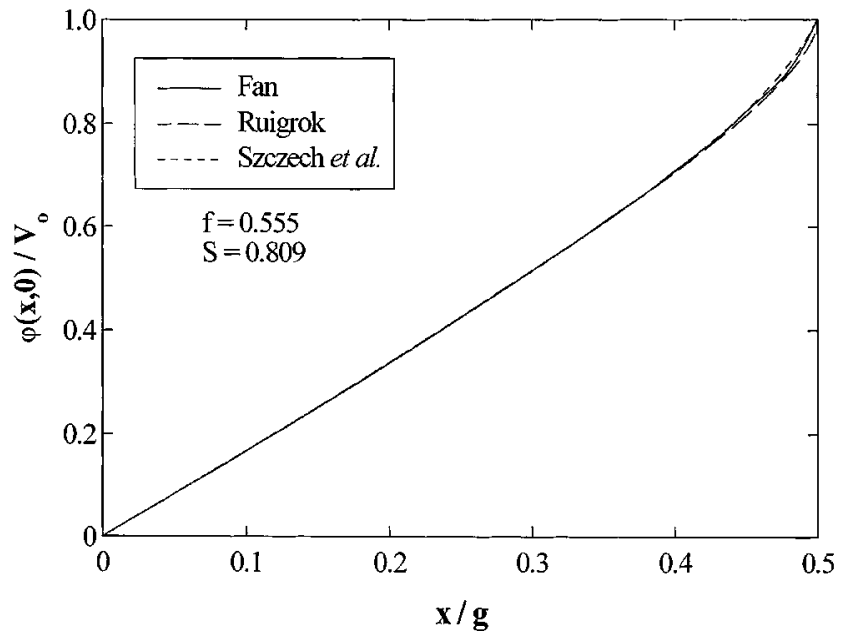

Fig. 1. The potential variation in the gap region for the Ruigrok and the Szczech et al. approximations and the exact Fan calculations ( $f=0.555, K=0.835, L=0.0433, M=0.52)$.

where the coefficients $B_{n}$ were found by integrating

$$
B_{n}=\frac{4}{g} \int_{x=0}^{g / 2} \tanh ^{-1}\left(\frac{x}{M g}\right) \cdot \sin \left(\frac{2 n \pi}{g} x\right) d x
$$

to give

$$
\begin{aligned}
B_{n}= & \frac{-1}{n \pi}\left\{2(-1)^{n} \tanh ^{-1}(1 /(2 M))+\cos (2 n \pi M)\right. \\
& \times[C i(n \pi(2 M-1))-C i(n \pi(2 M+1))]+\sin (2 n \pi M) \\
& \times[S i(n \pi(2 M-1))-\operatorname{Si}(n \pi(2 M+1))]\}
\end{aligned}
$$

$\mathrm{Ci}$ and $\mathrm{Si}$ are the cosine and sine integrals, respectively. It can be seen that the Fourier coefficients in (10) are dependent on the parameter $M$. $M$ was initially specified by Szczech et al. to be equal to 0.512 . In the following calculations, a value for $M$ equal to 0.52 (in agreement with the fittings of Iverson and Stubbs [9]) will be used as it was found that this value offers the lowest values of the correction terms and, hence, the closest approximation to the exact solution.

Equation (9) can be written in the form

$$
\begin{aligned}
\varphi(x, 0) \approx & \frac{2 V_{o}}{g} x+\frac{2 V_{o}}{g} x(K S-1) \\
& +\frac{2 L S V_{o}}{M} \sum_{n=1}^{\infty} B_{n} \sin \left(\frac{2 n \pi}{g} x\right)
\end{aligned}
$$

where the second term can be expanded into a Fourier series and a series of correction terms with coefficients $C_{n}^{1} / V_{o}$ can be added to make the potential exact. Then

$$
\begin{aligned}
\varphi(x, 0)= & \frac{2 V_{o}}{g} x+2 V_{o} \sum_{n=1}^{\infty} \\
& \times\left[\frac{L S}{M} B_{n}-\frac{(-1)^{n}}{n \pi}(K S-1)+\frac{C_{n}^{1}}{V_{o}}\right] \\
& \times \sin \left(\frac{2 n \pi}{g} x\right) .
\end{aligned}
$$

Matching (12) to the Fan potential in (1b), it can be shown that

$$
\frac{C_{n}^{1}}{V_{o}}=\frac{A_{n}}{V_{o}}-2\left[\frac{L S}{M} B_{n}-\frac{(-1)^{n}}{n \pi}(K S-1)\right] \text {. }
$$


TABLE II

HARMONIC COEFFICIENT VALUES FOR DifFerent VALUES OF $S$ FOR THE SZCZECH et al. APPROXIMATION ( $K=0.835, L=0.0433, M=0.52$ )

\begin{tabular}{c|c|c|c|c}
\hline $\mathbf{n}$ & $\mathbf{A}_{\mathbf{n}} / \mathbf{V}_{\mathbf{0}}$ & $\begin{array}{c}\mathbf{C}_{\mathrm{n}}^{\mathbf{1}} / \mathbf{V}_{\mathbf{o}} \\
\mathbf{S}=\mathbf{0 . 8 3}\end{array}$ & $\begin{array}{c}\mathbf{C}_{\mathbf{n}}^{\mathbf{1}} / \mathbf{V}_{\mathrm{o}} \\
\mathbf{S}=\mathbf{0 . 8 0 8 8 1 6 1 8 2}\end{array}$ & $\begin{array}{c}\mathbf{C}_{\mathbf{n}}^{1} / \mathbf{V}_{\mathbf{o}} \\
\mathbf{S}=\mathbf{0 . 7 9 7 8 5 7 4 2 5}\end{array}$ \\
\hline $\mathbf{1}$ & -0.08615711721 & -0.01737017376 & 0.00000000000 & 0.00934789995 \\
\hline 2 & 0.02915024465 & 0.00926654034 & 0.00014193729 & -0.00476854187 \\
\hline 3 & -0.01525421892 & -0.00712775589 & -0.00091280763 & 0.00243181721 \\
\hline 4 & 0.00959249954 & 0.00593991911 & 0.00122038799 & -0.00131946598 \\
\hline 5 & -0.00668032954 & -0.00511606338 & -0.00130908264 & 0.00073967494 \\
\hline 6 & 0.00496516208 & 0.00449297987 & 0.00130150642 & -0.00041601091 \\
\hline 7 & -0.00386089655 & -0.00400018532 & -0.00125217356 & 0.00022669127 \\
\hline 8 & 0.00310357777 & 0.00359924099 & 0.00118606217 & -0.00011260959 \\
\hline 9 & -0.00255909083 & -0.00326632182 & -0.00111497406 & 0.00004279119 \\
\hline 10 & 0.00215301133 & 0.00298549060 & 0.00104453891 & 0.00000000000 \\
\hline 11 & -0.00184115674 & -0.00274553997 & -0.00097737399 & -0.00002582112 \\
\hline 12 & 0.00159585983 & 0.00253830219 & 0.00091458445 & 0.00004076758 \\
\hline 13 & -0.00139902807 & -0.00235766061 & -0.00085650681 & -0.00004864871 \\
\hline 14 & 0.00123839761 & 0.00219893382 & 0.00080308987 & 0.00005190512 \\
\hline 15 & -0.00110540014 & -0.00205847372 & -0.00075409444 & -0.00005213215 \\
\hline 16 & 0.00099389255 & 0.00193339361 & 0.00070919945 & 0.00005038943 \\
\hline 17 & -0.00089937094 & -0.00182137886 & -0.00066805831 & -0.00004738950 \\
\hline 18 & 0.00081846814 & 0.00172055180 & 0.00063032835 & 0.00004361576 \\
\hline 19 & -0.00074862316 & -0.00162937342 & -0.00059568549 & -0.00003939794 \\
\hline 20 & 0.00068785821 & 0.00154657060 & 0.00056383076 & 0.00003496133 \\
\hline & & & &
\end{tabular}

Values of $C_{n}^{1} / V_{o}$ are shown in Table II for the values $L=$ $0.0433, M=0.52$, and $S=0.83$, as given by Szczech et al. It can be seen that the correction terms are larger than and decay more slowly than those of the Ruigrok expression. However, with $L=0.0433, M=0.52$, and $S=0.8088 \cdots$, the correction terms are of little significance and the corresponding fields and potentials are very good approximations to the exact. This is demonstrated in Fig. 1.

In a similar way to the calculation in the previous section, it is possible to select values of $S$ to make $C_{n}^{1}=0$ by solving (13). In this way, when $L=0.0433, M=0.52$, and $S=0.7978 \ldots$ $C_{10}^{1}=0$ and the other values of $C_{n}^{1}$ are as shown in Table II.

\section{GAP-Loss FunCtions AND HEAD FIELDS}

To further substantiate the claims about the accuracy made in the previous sections, the gap-loss functions for the various potentials are calculated and compared with the exact value from Fan. The gap-loss function is obtained from the Fourier transform of the head surface field and for the Fan distribution is [10]

$$
\begin{aligned}
\frac{H_{x}(k, 0)}{N I}= & \frac{\sin (k g / 2)}{(k g / 2)}+\left(\frac{k g \pi}{2}\right) \sum_{n=1}^{\infty} \\
& \cdot \frac{n(-1)^{n}\left(A_{n} / V\right) \sin (k g / 2)}{\left[(k g / 2)^{2}-(n \pi)^{2}\right]}
\end{aligned}
$$

whereas for the Ruigrok approximation of (2), it is [4], [6], [10]

$$
\frac{H_{x}(k, 0)}{N I} \approx(f) \frac{\sin (k g / 2)}{(k g / 2)}+(1-f) J_{\circ}(k g / 2) \text {. }
$$

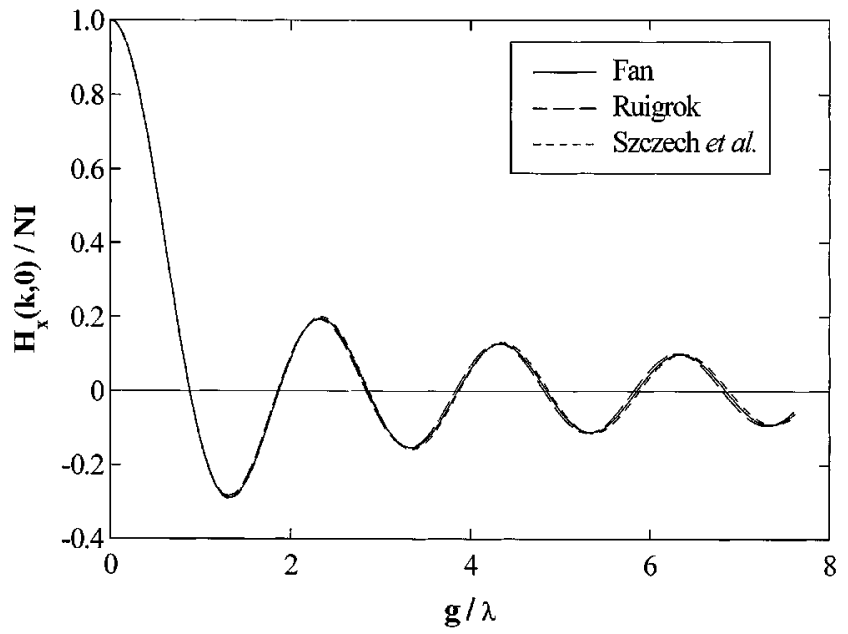

Fig. 2. Gap loss functions for Fan, Ruigrok with $f=0.555$ and Szczech et al. with $S=0.809(K=0.835, L=0.0433, M=0.52)$.

The surface field of Szczech et al. is given by [7]

$$
H_{x}(x, 0) \approx \frac{2 K S V_{o}}{g}+\frac{2 L S V_{o} g}{(M g)^{2}-x^{2}} \quad-g / 2 \leq x \leq g / 2
$$

which has a Fourier transform [4]

$$
\begin{aligned}
\frac{H_{x}(k, 0)}{N I} \approx & K S \frac{\sin (k g / 2)}{(k g / 2)}+\frac{L S}{M}\{\cos (k g M) \\
& \times\left[C i\left(\frac{k g}{2}(2 M+1)\right)-C i\left(\frac{k g}{2}(2 M-1)\right)\right] \\
& +\sin (k g M)\left[S i\left(\frac{k g}{2}(2 M+1)\right)\right. \\
& \left.\left.-S i\left(\frac{k g}{2}(2 M-1)\right)\right]\right\} .
\end{aligned}
$$

These gap-loss terms are plotted in Fig. 2 for $f=0.555$ and $L=0.0433, M=0.52$, and $S=0.809$. Their closeness to one another and to the exact result of Fan is obvious even as far as the seventh gap-null. In view of this and the smallness of the correction terms needed to make these modified Ruigrok and Szczech et al. expressions exact, it is clear that they are accurate enough for use in all but the most critical of applications. The implication is that corresponding field expressions given by Ruigrok [6], but with $f=0.555$, and by Szczech et al. [7] with $K=0.835, L=0.0433, M=0.52$, and $S=0.809$ are sufficiently accurate for all practical purposes.

\section{CONCLUSION}

The paper has shown that it is possible to take approximations to head fields or potential variations in the region of a head gap and add and evaluate an infinite series of correction terms that would make them exact. Further, it has been shown that by taking steps to reduce the correction terms, more accurate approximations to the exact fields can be obtained. The method has been used to show that the field expressions of Ruigrok and Szczech et al., which contain only one correction term, are very good approximations to the exact head fields, but that with minor modifications, they become extremely close to the exact head fields. 


\section{REFERENCES}

[1] G. J. Y. Fan, "A study of the playback process of a magnetic ring head," IBM J. Res. Dev., vol. 5, pp. 321-325, Oct. 1961.

[2] D. T. Wilton, B. K. Middleton, and M. Aziz, "Exact harmonic coefficients for a magnetic ring head," IEEE Trans. Magn., vol. 35, pp. 2043-2046, May 1999.

[3] O. Karlqvist, "Calculation of the magnetic field in the ferromagnetic layer of a magnetic drum," Trans. R. Inst. Technol., no. 86, pp. 3-27, 1954.

[4] J. J. M. Ruigrok, Short-Wavelength Magnetic Recording: New Methods and Analyses. Oxford: Elsevier, 1990.

[5] J. C. Mallinson, "Westmijze's 'thin' gap head revisited," IEEE Trans. Magn., vol. 26, pp. 3140-3154, Nov. 1990.
[6] G. A. Bertero, H. N. Bertram, and D. M. Barnett, "Fields and transforms for thin film heads," IEEE Trans. Magn., vol. 29, pp. 67-76, Jan. 1993.

[7] T. J. Szczech and P. R. Iverson, "An approach for deriving field equations for magnetic heads of different geometrical configurations," IEEE Trans. Magn., vol. 22, pp. 355-360, Sept. 1986.

[8] — - "Improvement of the coefficients in field equations for thin-film recording heads," IEEE Trans. Magn., vol. MAG-23, pp. 3866-3867, Sept. 1987.

[9] P. R. Iverson and D. P. Stubbs, "Effects of saturation on ring head fields," IEEE Trans. Magn., vol. 27, pp. 4897-4899, Nov. 1991.

[10] C. D. Mee, The Physics of Magnetic Recording. Amsterdam: NorthHolland, 1964.

[11] W. K. Westmijze, "Studies on magnetic recording," Philips Res. Rep., Part II, vol. 8, pp. 161-183, June 1953. 